\title{
ESTADO NUTRICIONAL E CAPACIDADE FUNCIONAL EM IDOSOS HOSPITALIZADOS
}

Luana Constantinov Jamarino ${ }^{1}$, Michele Darlin Seregati ${ }^{1}$, Stéphane Raquel Almeida Velande da Fonseca ${ }^{2}$, Daniele Fernanda Felipe ${ }^{3}$, Ariana Ferrari $^{3}$

${ }^{1}$ Graduada em Nutrição na Universidade Cesumar - UNICESUMAR, Maringá-PR, Brasil

${ }^{1}$ Mestranda do Programa de Pós-graduação em Ciência, Tecnologia e Segurança Alimentar da Universidade Cesumar - UNICESUMAR, Maringá-PR, Brasil

${ }^{3}$ Docente do Programa de Pós-graduação em Tecnologias Limpas da Universidade Cesumar - UNICESUMAR, Maringá-PR, Brasil

E-mail: ariana.ferrari@unicesumar.edu.br

Recebido em: 15/08/2020 - Aprovado em: 15/09/2020 - Publicado em: 30/09/2020 DOI: 10.18677/EnciBio_2020C20

O envelhecimento é condição natural dos humanos, nele evidenciam-se diversas transformações. Objetivou-se analisar o estado nutricional e a capacidade funcional de idosos hospitalizados no Hospital Municipal de Maringá-PR. Para avaliar o estado nutricional (EN) foram utilizados parâmetros como o índice de massa corporal (IMC), prega cutânea tricipital (PCT), circunferência do braço (CB), circunferência muscular do braço $(\mathrm{CMB})$ e área muscular do braço corrigida $(\mathrm{AMBc})$. Para avaliar a capacidade funcional foi utilizado o Índice Katz. Participaram da pesquisa 130 pacientes o qual verificou-se que a maioria estava com baixo peso para o IMC (64,2\% das mulheres e 50,8\% dos homens). Quanto a CB, as porcentagens de homens classificados em EN adequado, ou em baixa reserva ficam próximas de $41,2 \%$ e $36,5 \%$, respectivamente. Resultados similares foram encontrados nas mulheres, que foram classificadas em baixa reserva em 34,3\%, risco de déficit em $25,4 \%$ e adequado em $35,8 \%$ do total de mulheres da pesquisa. Ao analisar a PCT e a AMBc os avaliados de ambos os sexos foram predominantemente classificados em adequado $(71,4 \%$ e $50,8 \%$ dos homens e $44,8 \%$ e $64,2 \%$ das mulheres, respectivamente). Já na avaliação da $\mathrm{CMB}, 46 \%$ dos homens, foram classificados como baixa reserva e $59,6 \%$ das mulheres como adequado. Para a capacidade funcional, os idosos foram classificados como muito dependente para ambos os gêneros $(61,9 \%$ dos homens e $61,2 \%$ das mulheres). Conclui-se que alterações do estado nutricional e da capacidade funcional de idosos é comum em ambiente hospitalar. Portanto, faz-se necessário que a avaliação desses parâmetros seja realizada em rotina nutricional a fim de prevenir o declínio das condições clínicas dos idosos.

PALAVRAS-CHAVE: antropometria, desnutrição, envelhecimento. 


\title{
NUTRITIONAL STATUS AND FUNCTIONAL CAPACITY IN HOSPITALIZED ELDERLY
}

\begin{abstract}
Aging is a natural condition of humans, in which there are several transformations. The objective of this study was to analyze the nutritional status and functional capacity of elderly hospitalized at the Municipal Hospital of Maringá-PR. To assess nutritional status (EN), parameters such as body mass index (BMI), tricipital skinfold (PCT), arm circumference (CB), arm muscle circumference (CMB) and corrected arm muscle area (AMBc) were used ). To assess functional capacity, the Katz Index was used. 130 patients participated in the research, which found that most were underweight for BMI (64.2\% of women and $50.8 \%$ of men). As for CB, the percentages of men classified in adequate $E N$, or in low reserve are close to $41.2 \%$ and $36.5 \%$, respectively. Similar results were found in women, who were classified as low reserve in $34.3 \%$, risk of deficit in $25.4 \%$ and adequate in $35.8 \%$ of the total women in the survey. When analyzing the PCT and AMBc, those evaluated for both sexes were predominantly classified as adequate $(71.4 \%$ and $50.8 \%$ of men and $44.8 \%$ and $64.2 \%$ of women, respectively). In the CMB assessment, $46 \%$ of men were classified as low reserve and $59.6 \%$ of women as adequate. For functional capacity, the elderly were classified as very dependent for both genders $(61.9 \%$ of men and $61.2 \%$ of women). It is concluded that changes in the nutritional status and functional capacity of the elderly are common in a hospital environment. Therefore, it is necessary that the evaluation of these parameters be carried out in a nutritional routine in order to prevent the decline in the clinical conditions of the elderly.
\end{abstract}

KEYWORDS: anthropometry, malnutrition, aging.

\section{INTRODUÇÃO}

O envelhecimento é uma condição natural da espécie humana, sendo dinâmico e progressivo, no qual, são evidenciadas mudanças, tais como um acentuado declínio da natalidade, e simultaneamente, uma redução da mortalidade e do aumento da longevidade, oriundo dos avanços nos cuidados em saúde (SILVA et al., 2019).

Segundo Shuhada et al. (2017), a projeção de crescimento populacional para o ano de 2030, é de 1,4 bilhões, sendo que, no ano de 2050, poderá chegar a 2,1 bilhões. Em paralelo a isso, a expectativa de vida da população brasileira aumentou consideravelmente nas últimas décadas. Segundo Camargo et al. (2019), no ano de 1940 ao atingir 60 anos, um indivíduo vivia em média 13,2 anos, 11,6 anos para os homens e 14,5 anos para as mulheres. Já no ano de 2014, esses dois últimos valores foram acrescidos de 8,4 e 9,1 anos, respectivamente.

Miranda et al., (2016) indicam que em 2050 a população brasileira será de aproximadamente de 253 milhões de habitantes, a quinta maior população do planeta. Partindo do pressuposto que idosos são pessoas com 60 anos ou mais de idade, até 2050 , o percentual de idosos se duplicará (de $11 \%$ para $22 \%$ ), sendo que no caso dos muito idosos, ou seja, aqueles com idade superior a 80 anos, a porcentagem quadruplicará (MIRANDA et al., 2016; KATSAS et al., 2019).

Conforme Oliveira et al. (2018), de 2001 a 2004, os idosos representavam $8,4 \%$ da população brasileira, e a taxa de hospitalização era 13\%, entretanto, no ano 2009 , os idosos foram responsáveis por $21 \%$ das internações no Brasil. Dado importante é que mais da metade das admissões em Unidade de Terapia Intensiva (UTI) são de indivíduos com 65 anos ou mais, sendo essa vulnerabilidade provocada 
pelos eventos agudos ameaçadores da vida, bem como a necessidade de cuidados intensivos (DIETRICH et al., 2017).

Freitas et al. (2017) definem a vulnerabilidade como a capacidade de um indivíduo sofrer dano em resposta a um estímulo, tanto a nível biomédico, como nos aspectos sociais e econômicos. Sabe-se que o aumento do envelhecimento vem acompanhado com o aumento de comorbidades, como por exemplo as doenças crônicas não transmissíveis (DCNT) (CHAN et al., 2019). Além das mudanças nos aspectos psicológicos e sociais, há grandes mudanças fisiológicas e metabólicas no processo do envelhecimento que, consequentemente, provocam alterações físicas, como perda de mobilidade. Tais modificações podem acarretar em comprometimento do grau de dependência do idoso com modificações no estado nutricional (CENDRON et al., 2003).

Conforme Barbosa et al. (2019) o estado nutricional é conceituado pelo resultado entre a ingestão de energia, nutrientes e micronutrientes e as necessidades do organismo, bem como, pela capacidade de digestão, absorção, utilização dos nutrientes e a interação de fatores patológicos. A Sociedade Americana de Nutrição Enteral e Parenteral (SBNPE) relata que a diminuição do metabolismo associado a um desequilíbrio entre 0 aporte nutricional $e$ as necessidades do indivíduo, são fatores determinantes que aumentam o risco do desenvolvimento de desnutrição. Foi observado, por exemplo, que $30 \%$ dos pacientes hospitalizados tornavam-se desnutridos nas primeiras 48 horas de internação, e ainda, durante um intervalo de tempo de três a sete dias esse percentual elevava-se em $15 \%$, alcançando em torno de $60 \%$ após quinze dias de internação (LEITE et al., 2005). Nesse sentido, Bhirommuang et al. (2019) concluem que, aproximadamente, $50 \%$ dos pacientes hospitalizados são identificados como desnutridos.

A desnutrição detectada na admissão hospitalar possui etiologia primária, causada pela ingestão inadequada e insatisfatória de nutrientes. Essa condição nutricional pode ocorrer em virtude de condições socioeconômicas difíceis que não garantem a reposição energético-protéica e dos demais nutrientes necessários ou secundariamente por fatores intrínsecos, devido ao impacto da doença (TEIXEIRA et al., 2016). Verifica-se ainda que, problemas na deglutição e mastigação, depressão, isolamento social e insuficiência cardíaca são considerados as causas principais da desnutrição em idosos (LOURO, 2004).

Segundo Leite et al. (2005) indivíduos que apresentam infecções graves, traumatismos ou que estão em pós-operatório de grandes cirurgias são mais vulneráveis a desenvolver desnutrição. Os autores ainda ressaltam que a ingestão diminuída, restrição de oferta hídrica, instabilidade hemodinâmica, diminuição da absorção e interação droga-nutriente também afetam o estado nutricional. Nota-se ainda que, durante o período de internação hospitalar, intrinsicamente os indivíduos diminuem a ingestão de energia, em virtude da perda de apetite, restrição alimentar oral inadequada, disfagia e má absorção relacionadas à doença. Além disso, a presença de infecções, processos inflamatórios, estresse e distúrbios metabólicos podem desencadear ou até acelerar o quadro de desnutrição (OLIVEIRA et al., 2017; BHIROMMUANG et al., 2019; KATSAS et al., 2019).

Outra questão que pode ser observada em idosos é a perda da capacidade funcional. Segundo Silva et al., (2018), as condições físicas e mentais responsáveis por manter uma vida ativa e independente, na qual o indivíduo consegue manter seus hábitos e realizar suas ações diárias sem ajuda ou dependência de outra pessoa, são definidas como capacidade funcional. Alterações nesse parâmetro 
podem levar a maiores prejuízos na saúde e estado nutricional dos idosos (CENDRON et al., 2003). Além disso, a hospitalização é considerada um fator de risco, principalmente para pacientes geriátricos, já que, influencia na redução da capacidade funcional e na qualidade de vida, as quais podem ser irreparáveis (OLIVEIRA et al., 2018).

Partindo do pressuposto que alterações do estado nutricional e da capacidade funcional de idosos pode levar a uma série de prejuízos na saúde e piora da qualidade de vida (NAJAS ; YAMATTO, 2008), a avaliação do consumo alimentar, da composição corporal e dos dados imunológicos tornam-se importantes ferramentas para a identificação de problemas associados a saúde desses indivíduos (LOURO, 2004). Além disso, para que a avaliação nutricional do idoso seja realizada de maneira precisa e adequada, os parâmetros bioquímicos, clínicos, dietéticos e antropométricos são de extrema importância (TAVARES et al., 2015).

As medidas antropométricas recomendadas para usar em idosos são: o peso, a estatura, a circunferência do braço (CB) - que indica a reserva energética e protéica do idoso - e algumas pregas cutâneas, necessárias para estimar a massa magra e a massa gorda desses indivíduos (ACUÑA ; CRUZ, 2004; TAVARES et al., 2015). O Índice de Massa Corpórea (IMC) é uma medida secundária calculada através da divisão do peso pela estatura ao quadrado, sendo classificado segundo os valores de referências para idosos (NAJAS; YAMATTO, 2008).

Desse modo, apesar de existir diversos métodos para a investigação da desnutrição em pacientes hospitalizados essas avaliações não são realizadas de forma frequente, contribuindo para que as consequências provenientes da internação se estendam, tornando o tempo de permanência do idoso no ambiente hospitalar maior e, consequentemente, contribuindo para o aumento dos índices de mortalidade (LOURO, 2004; TEIXEIRA et al., 2016).

Portanto, o objetivo dessa pesquisa foi avaliar o estado nutricional e a capacidade funcional de idosos hospitalizados em um hospital municipal do interior do Paraná.

\section{MATERIAIS E METODOS}

Trata-se de um estudo descritivo e transversal, com coleta prospectiva de dados, realizada no Hospital Municipal na cidade de Maringá-PR, no período de novembro de 2017 a maio de 2018. Foram avaliados idosos internados no setor de clínica médica, setor este composto por 30 leitos. A definição da amostra foi realizada de maneira aleatória e não probabilística.

Foram adotados como critérios de inclusão: idosos internados em um hospital municipal na cidade de Maringá-PR, que aceitassem participar do estudo, assinassem o termo de consentimento e colaborassem com a coleta de dados antropométricos. Foram excluídos os pacientes que não apresentavam condições clínicas para a avaliação do estado nutricional. Duas estagiárias do último ano do curso de nutrição da Universidade Cesumar (Unicesumar) foram responsáveis pela coleta de dados, sob a orientação da supervisora de estágio.

As avaliações foram realizadas de segundas a sextas-feiras. Os dados sociodemográficos coletados foram o sexo e a idade. Para avaliar o estado nutricional do idoso hospitalizado foi aferido o peso, a estatura, a prega cutânea triciptal (PCT) e a CB. O peso foi medido utilizando uma balança de plataforma digital da marca G-Tech®. A aferição da estatura do paciente foi realizada com o mesmo, em pé, descalço, com os calcanhares e pés unidos, panturrilhas encostadas na parede, em posição ereta, olhando para frente (plano horizontal de Frankfort), 
através do estadiômetro portátil da marca Welmyß. Em pacientes que não foi possível a aferição do peso e altura realizou-se estimativa na forma da equação proposta por Chumlea et al. (1988).

Para o cálculo do IMC foi utilizada a fórmula: Peso atual $(\mathrm{kg}) /(\text { Altura })^{2}(\mathrm{~m})$. A classificação foi realizada pelos pontos de corte recomendados pela Organização Pan-Americana de Saúde (OPAS, 2002): baixo peso (IMC $\leq 23 \mathrm{~kg} / \mathrm{m}^{2}$ ), peso adequado (IMC $>23$ e $<28 \mathrm{~kg} / \mathrm{m}^{2}$ ), risco para obesidade ( IMC $\geq 28$ e $<30 \mathrm{~kg} / \mathrm{m}^{2}$ ) e obesidade (IMC $\geq 30 \mathrm{~kg} / \mathrm{m}^{2}$ ). A PCT foi classificada através da tabela de classificação dos idosos, segundo percentis para ambos os gêneros. Depois de identificado o percentil, a PCT foi classificada em depleção (percentil $<5$ ), risco para déficit (percentil entre 5,1 e 15), adequado (entre o percentil 15,1 e 85), acima da média (entre o percentil 85,1 e 95) e excesso de gordura (percentil maior ou igual a 95) (FRISANCHO, 1990).

A CB foi classificada através da tabela de classificação dos idosos, segundo percentis para ambos os gêneros. Depois de identificado o percentil, a $C B$ foi classificada segundo Frisancho et al., (1990). A CMB foi calculada a partir da equação proposta por Gurney e Jelliffe (1973): CMB $(\mathrm{cm})=$ CB $(\mathrm{cm})-\pi \times($ PCT $(\mathrm{mm})$ $\div$ 10). Em seguida, foi classificada através da tabela de classificação dos idosos segundo percentis para ambos os gêneros e posterior análise do percentil, sendo a CMB classificada em depleção (percentil <5), risco de déficit (percentil entre 5,1 e 15), adequado (entre o percentil 15,1 e 85), acima da média (entre o percentil 85,1 e 95) e aumento da massa muscular (percentil maior ou igual a 95) (FRISANCHO, 1990).

Para o cálculo da AMBc foram utilizadas fórmulas propostas por Heymsfield et al. (1982). Depois de calculada, os valores de AMBc foram comparados à tabela dos idosos segundo percentis para gênero masculino e feminino e classificados em depleção (percentil $\leq 5$ ), risco de déficit (percentil entre 5,1 e 15), adequado (percentil entre 15,1 e 85), acima da média (percentil entre 85,1 e 95) e aumento de massa muscular (percentil $\geq 95$ ) (FRISANCHO, 1990).

A Avaliação da Capacidade Funcional foi realizada a partir do Índice de Katz, elaborado em 1963, por Sidney Katz e colaboradores, que é utilizado mundialmente com o objetivo de avaliar a capacidade funcional em idosos (KATZ et al., 1963). No presente trabalho, para avaliar a funcionalidade para Atividades Básicas de Vida Diária (ABVDs) foi utilizado o Índice de Katz, modificado por The Hartford Institute for Geriatric Nursing (1998), que é composto de seis parâmetros: capacidade para tomar banho, se vestir, ir ao banheiro, transferência, continência e alimentação. A pontuação varia de zero a seis, sendo que é atribuído um ponto para cada atividade que o idoso realiza sem precisar de ajuda. Após a análise dos pontos atribuídos, foi realizado o somatório dos pontos e os idosos foram classificados em três categorias: independência ( $\geq 6$ pontos); dependência parcial/moderada ( 3 e 5 pontos) e dependência severa ( $\leq 2$ pontos) (HARTFORD INSTITUTE FOR GERIATRIC NURSING, 1998).

A idade, peso e estatura dos idosos foram analisados de forma descritiva através de média e desvio-padrão para retratar a variação e a representatividade do conjunto amostral. Para cada uma das variáveis indicadoras de estado nutricional foi testada a diferença entre os três níveis de classificação do Índice de Katz (Independente, Dependência moderada e Muito dependente) através de Análises de Variância unifatorial (one-way ANOVA), seguidas do Teste de Tukey para comparálos par-a-par sob nível de significância de $5 \%$. Os pressupostos de normalidade e homocedasticidade da ANOVA foram testados pelos testes de Shapiro-Wilks e 
Levene, respectivamente. Todas as variáveis alcançaram esses pressupostos sem necessidade de transformação. As análises foram realizadas no software Statistica10 (STATSOFT, 2011).

A coleta de dados foi realizada após a aprovação do Comitê de Ética em Pesquisa, com protocolo sob n. 2.496.830.

\section{RESULTADOS E DISCUSSÃO}

A amostra foi constituída com um total de 154 pacientes, sendo 78 do sexo masculino $(50,65 \%)$ e 76 do sexo feminino $(49,35 \%)$. A idade dos idosos variou entre 60 e 103 anos (média $=77,60$, desvio-padrão $=10,17$ ). As variáveis nutricionais e suas respectivas classificações estão representadas na Tabela 1.

Alterações do estado nutricional em pacientes hospitalizados, atua diretamente na evolução clínica (BARBOSA et al., 2019). Em relação ao IMC, quase $60 \%$ dos idosos estavam com baixo peso, sendo que entre as mulheres, aproximadamente, $67 \%$ encontravam-se abaixo do peso recomendado para a idade. Esses resultados são similares aos encontrados por Panissa e Vassimon (2012), no qual $41 \%$ dos idosos avaliados na Santa Casa de Franca estavam desnutridos, 33\% em eutrofia e $25 \%$ deles com excesso de peso. Resultados semelhantes foram encontrados no estudo realizado por Zanchim et al. (2013), em que a prevalência de desnutrição nos idosos hospitalizados foi alta.

Sabe-se que a desnutrição é um fator predisponente para enfermidades e está associada a maior tempo de permanência no ambiente hospitalar e mortalidade, devendo ser identificada e tratada de forma precoce pela equipe multiprofissional, a fim de reduzir gastos em serviços de saúde, proporcionar uma recuperação mais rápida, melhor qualidade de vida do paciente e inibição das complicações associadas às doenças (AZEVEDO et al., 2007). Além disso, essa condição nutricional pode levar a atrofia e redução da massa muscular, a diminuição respiratória e cardíaca, a perda da função muscular, a má absorção de micronutrientes e macronutrientes, como cálcio e zinco, o aumento do risco de tromboembolismo, o mau funcionamento renal, a fadiga, a anorexia e a deficiência do sistema imunológico (MISHAMANDANI et al., 2019). Ainda, conforme Silva et al. (2019), os idosos desnutridos podem apresentar problemas neurológicos, sarcopenia, perda da flexibilidade, massa óssea, equilíbrio, funções locomotoras e cardiovasculares.

TABELA 1. Estado nutricional dos idosos segundo as variáveis nutricionais de pacientes idosos do Hospital Municipal de Maringá, de acordo com o sexo.

\begin{tabular}{|l|c|c|c|}
\hline Variáveis e classificações nutricionais & $\begin{array}{c}\text { Total } \\
\mathbf{n}(\%)\end{array}$ & $\begin{array}{c}\text { Masculino } \\
\mathbf{n}(\%)\end{array}$ & $\begin{array}{c}\text { Feminino } \\
\mathbf{n}(\%)\end{array}$ \\
\hline IMC & & & \\
\hline Baixo peso & $92(59,74)$ & $41(52,56)$ & $51(67,11)$ \\
\hline Peso adequado & $41(26,62)$ & $23(29,49)$ & $18(23,68)$ \\
\hline Risco para obesidade & $8(5,19)$ & $5(6,41)$ & $3(3,95)$ \\
\hline Obesidade & $13(8,44)$ & $9(11,54)$ & $4(5,26)$ \\
\hline CB & & & \\
\hline Depleção/baixa reserva & $56(36,36)$ & $30(38,46)$ & $26(34,21)$ \\
\hline Abaixo da média/risco de déficit & $34(22,08)$ & $15(19,23)$ & $19(25,00)$ \\
\hline Média/adequado & $58(37,66)$ & $30(38,46)$ & $28(36,84)$ \\
\hline Acima da média/adequado & $4(2,60)$ & $2(2,56)$ & $2(2,63)$ \\
\hline Aumento de massa muscular/boa nutrição & $2(1,30)$ & $1(1,28)$ & $1(1,32)$ \\
\hline
\end{tabular}




\begin{tabular}{|l|c|c|c|}
\hline PCT & & & \\
\hline Depleção/baixa reserva & $20(12,99)$ & $4(5,13)$ & $16(21,05)$ \\
\hline Abaixo da média/risco de déficit & $36(23,38)$ & $11(14,10)$ & $25(32,89)$ \\
\hline Média/adequado & $87(56,49)$ & $52(66,67)$ & $35(46,05)$ \\
\hline Acima da média/adequado & $10(6,49)$ & $10(12,82)$ & $0(0,00)$ \\
\hline Aumento de massa muscular/boa nutrição & $1(0,65)$ & $1(1,28)$ & $0(0,00)$ \\
\hline CMB & & & \\
\hline Depleção/baixa reserva & $54(35,06)$ & $37(47,44)$ & $17(22,37)$ \\
\hline Abaixo da média/risco de déficit & $26(16,88)$ & $17(21,79)$ & $9(11,84)$ \\
\hline Média/adequado & $65(42,21)$ & $20(25,64)$ & $45(59,21)$ \\
\hline Acima da média/adequado & $6(3,90)$ & $3(3,85)$ & $3(3,95)$ \\
\hline Aumento de massa muscular/boa nutrição & $3(1,95)$ & $1(1,28)$ & $2(2,63)$ \\
\hline AMBc & & & \\
\hline Depleção/baixa reserva & $19(12,34)$ & $11(14,10)$ & $8(10,53)$ \\
\hline Abaixo da média/risco de déficit & $28(18,18)$ & $21(26,92)$ & $7(9,21)$ \\
\hline Média/adequado & $90(58,44)$ & $39(50,00)$ & $51(67,11)$ \\
\hline Acima da média/adequado & $9(5,84)$ & $2(2,56)$ & $7(9,21)$ \\
\hline Aumento de massa muscular/boa nutrição & $8(5,19)$ & $5(6,41)$ & $3(3,95)$ \\
\hline
\end{tabular}

Apesar da CB estar adequada em $38,46 \%$ dos homens e $36,84 \%$ das mulheres, a quantidade de idosos do sexo masculino abaixo da média ou com depleção foi $57,69 \%$. Já entre as mulheres, 59,21\% apresentavam-se com depleção ou risco para déficit em relação a CB. Souza et al. (2018), ao avaliarem o estado nutricional dos pacientes hospitalizados, encontraram uma prevalência de eutrofia, tanto no público adulto quanto nos idosos em relação a CB, contudo, uma parcela apresentou desnutrição, variando de leve, moderado a grave. Todavia, os autores relatam que, conforme o estado nutricional é depletado ou preservado, as médias de IMC, circunferência do braço e da panturrilha acompanham o nível do estado nutricional.

A aferição da espessura da PCT permite estimar a gordura corporal dos pacientes (WOODROW, 2009). No presente trabalho, a maior parte dos avaliados encontravam-se adequados em relação a PCT. Segundo Ribeiro et al. (2019), devido a alterações no metabolismo relacionadas à própria idade, além das mudanças na massa muscular, há também um aumento na gordura corporal total além de infiltração lipídica no músculo esquelético.

Em relação as variáveis indicativas de reserva de massa muscular (CMB e AMBc), a maioria das mulheres apresentavam-se com adequada massa muscular. Entretanto, aproximadamente, $34 \%$ e $20 \%$ das idosas encontravam-se com algum grau de déficit de reserva muscular segundo o $\mathrm{CMB}$ e $\mathrm{AMBc}$, respectivamente. Já entre os homens a perda de massa muscular foi evidenciada em mais de $69 \%$ segundo o CMB e em aproximadamente $41 \%$ de acordo com o AMBc. Os dados aqui encontrados se assemelham a um recente trabalho realizado com idosos admitidos em um setor de emergência, clínica médica e cirúrgica de um hospital público da Região Metropolitana de Recife. Neste estudo aproximadamente $50 \%$ dos idosos estavam desnutridos de acordo com o AMBc (ARRUDA et al., 2020). Kim e Choi (2015), demonstraram que existe uma associação entre a baixa massa muscular e rigidez arterial, que é um preditor independente para as doenças cardiovasculares. Além disso, déficits no IMC e AMBc foi inversamente associado a síndrome da fragilidade, caracterizada pela redução da reserva homeostática 
podendo aumentar o risco de quedas e fraturas, além de maior tempo de internação hospitalar e incapacidade (SAMPAIO et al., 2017).

De acordo com a avaliação da capacidade funcional, 97 idosos, ou seja, aproximadamente $63 \%$ de todos os pacientes, possuíam alto grau de dependência, sendo classificados como muito dependentes (Gráfico 1). Entre as mulheres e homens, quase $74 \%$ e $67 \%$ apresentavam algum grau de dependência, respectivamente. Esses dados se assemelham ao estudo de Cruz et al., (2011), aonde foi averiguado que aproximadamente $80 \%$ dos idosos que deram entrada no Hospital Santa Rosa em Cuiabá-MT possuíam capacidade funcional reduzida ou limitada.

GRÁFICO 1. Capacidade funcional dos idosos avaliados segundo Índice Katz.

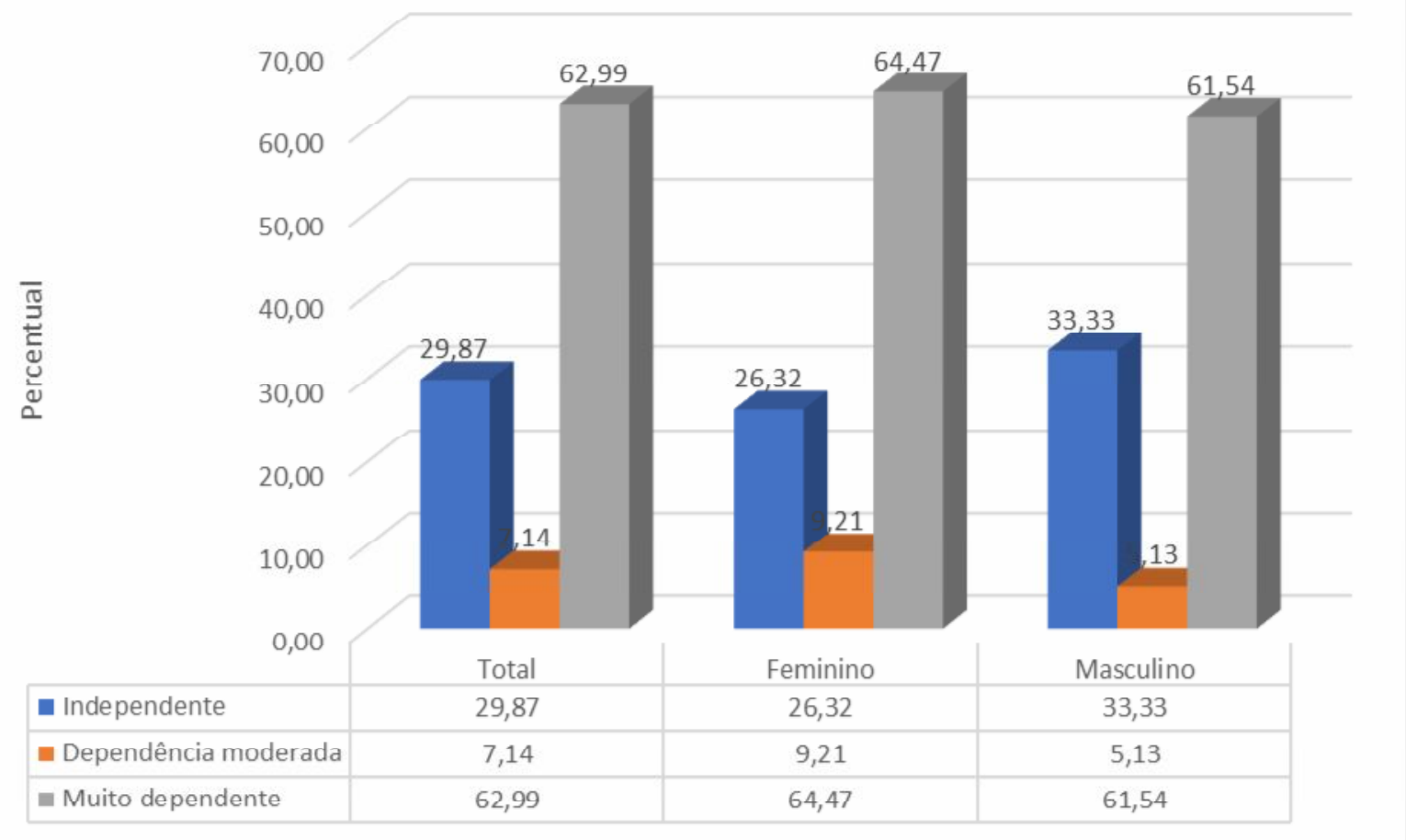

Fonte: As autoras, (2018).

Sabe-se que uma menor capacidade funcional predispõe o idoso ao surgimento de doenças e à ingestão insuficiente de nutrientes (CENDRON et al., 2003). Além disso, essas alterações podem aumentar as chances de quedas entre os idosos e, consequentemente, aumento das taxas de internação e mortalidade (ABREU et al., 2018).

As ANOVA indicaram diferença significativa entre os níveis do Índice de Katz para IMC (Figura 2A, $p<0,001$ ), CB (Figura 2B, $p<0,001$ ), CMB (Figura 2D, $p$ $<0,001$ ), AMBc (Figura 2E, $p<0,001$ ) e peso estimado dos acamados (Figura $2 \mathrm{~F}, p$ $<0,001$ ), mas não para PCT (Figura 2C, $p=0,22$ ) (Gráfico 2). 
GRÁFICO 2. Análises de Variância unifatorial (one-way ANOVA) entre os níveis (Independente, Dependência moderada e Muito dependente) do Índice de Katz para as variáveis indicadoras do estado nutricional de pacientes idosos do Hospital Municipal de Maringá, onde A- Índice de Massa Corporal (IMC); B - Circunferência do Braço (CB); C - Prega Cutânea Triciptal (TCP); D - Circunferência Muscular do Braço (CMB); E - Área Muscular do Braço corrigida (AMBc); F - peso estimado.
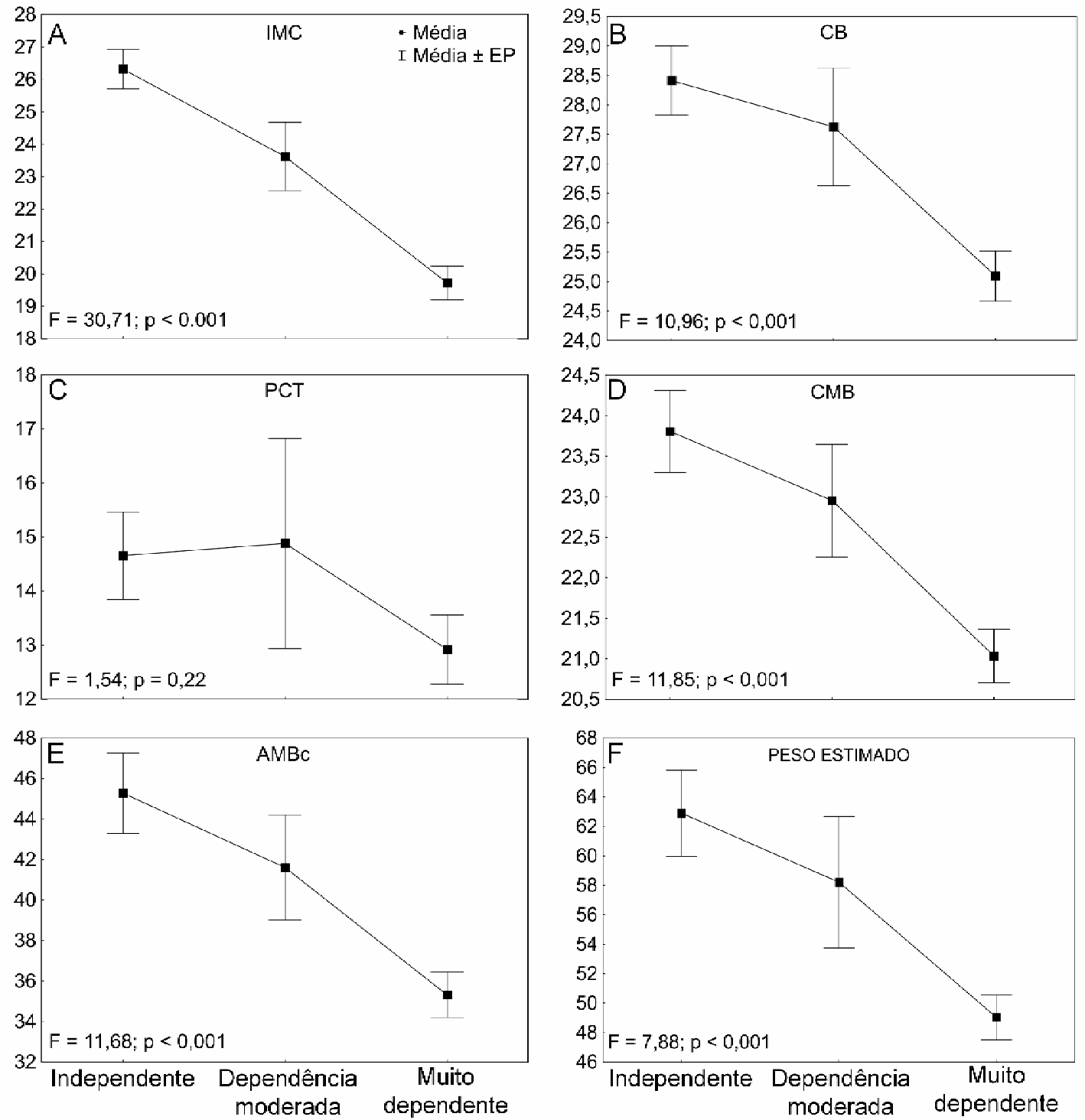

Indice de Katz

Fonte: As autoras, (2018).

Os testes de Tukey sobre os níveis do Índice de Katz relevaram diferenças significativas para IMC, entre Independente e Muito dependente $(p<0,001)$ e entre Dependência moderada e Muito dependente $(p=0,03)$, para CB, entre Independente e Muito dependente $(p<0,001)$ e para CMB, AMBc e peso estimado, entre Independente e Muito dependente $(p<0,001)$ (Tabela 2). As demais comparações par-a-par não velaram diferenças significativas segundo o Teste de Tukey. 
TABELA 2. Valores de significância $(p)$ do Teste de Tukey entre os níveis (Independente, Dependência moderada e Muito dependente) do Índice de Katz para as variáveis indicadoras de estado nutricional de pacientes idosos do Hospital Municipal de Maringá.

\begin{tabular}{lccc}
\hline $\begin{array}{l}\text { Variáveis } \\
\text { estado nutricional }\end{array}$ & $\begin{array}{c}\text { do } \\
\text { Independente } \mathbf{x} \\
\text { Dependência } \\
\text { moderada }\end{array}$ & $\begin{array}{c}\text { Independente } \mathbf{x} \\
\text { Muito dependente }\end{array}$ & $\begin{array}{c}\text { Dependência } \\
\text { moderada x Muito } \\
\text { dependente }\end{array}$ \\
\hline IMC & 0,21 & $<0,001^{*}$ & $0,003^{*}$ \\
CB & 0,83 & $<0,001^{*}$ & 0,12 \\
PCT & 0,99 & 0,25 & 0,57 \\
CMB & 0,71 & $<0,001^{*}$ & 0,15 \\
AMBc & 0,62 & $<0,001^{*}$ & 0,21 \\
Peso estimado & 0,76 & $<0,001^{*}$ & 0,18 \\
\hline
\end{tabular}

Desse modo, os resultados indicaram que o estado nutricional, como revelado pelas diferenças significativas encontradas para IMC, $C B, C M B, A M B c$ e peso estimado dos acamados, influencia na autonomia dos idosos. No trabalho desenvolvido por Cendron et al., (2003) foi verificada que a maioria dos idosos hospitalizados possuíam risco de desnutrição ou diagnóstico de desnutrição e dependência funcional. Segundo Azevedo et al., (2007), o estado nutricional em idosos possui alto grau de relevância, tendo em vista que as consequências da desnutrição, as variações do metabolismo, a ingestão de medicamentos e a menor ingestão de micronutrientes associados ao psicológico e à condição social e econômica, podem reduzir e influenciar negativamente a capacidade funcional do paciente.

\section{CONCLUSÃO}

A partir do presente estudo, constatou-se alterações do estado nutricional. Através da avaliação antropométrica notou-se que, independente do gênero, a incidência de desnutrição e seu risco estava elevada. A comparação de cada parâmetro antropométrico de maneira individualizada, bem como o IMC, demonstrou que a maioria dos idosos estavam com baixo peso. No entanto, de acordo com CB a porcentagem de homens classificados em estado nutricional adequado ou em depleção/baixa reserva foi bem próxima, e o mesmo aconteceu com as mulheres, na qual, a porcentagem das que foram classificadas em depleção muscular, risco de déficit ou adequada eram semelhantes.

Ao analisar PCT e AMBc os avaliados de ambos os sexos, eram predominantemente classificados em adequado. Já ao avaliar a CMB, os homens em sua maioria apresentam depleção muscular, enquanto que as mulheres, estado nutricional adequado. Quanto à capacidade funcional os idosos hospitalizados, estes apresentaram uma perda de função, os quais em sua maioria, foram classificados como muito dependentes. Partindo do pressuposto que o estado nutricional é importante para o desfecho clínico do paciente idoso é de vital importância que sejam realizadas avaliações periódicas dessas variáveis. Através do diagnóstico nutricional é possível estabelecer um plano precoce de atenção alimentar e nutricional para o paciente auxiliando assim, na saúde desses pacientes.

Uma possível limitação do presente trabalho refere-se ao fato de não ter sido avaliado a patologia e motivo de internamento dos pacientes idosos, o que pode interferir no estado nutricional e capacidade funcional. Além disso, não foi verificado 
associações estatísticas em nenhuma das análises. Assim, é necessário que mais estudos sejam realizados nessa área a fim se conhecer melhor o estado nutricional dos idosos hospitalizados além de verificar se esse estado nutricional influencia na incapacidade funcional.

\section{REFERÊNCIAS}

ABREU, D. R. DE O.M.; NOVAES, E. S.; de OLIVEIRA, R.R.; et al. Internação e mortalidade por quedas em idosos no Brasil: análise de tendência. Ciência \& Saúde Coletiva, v. 23, n.4, p.1131-1141, 2018. Disponivel em: $<$ http://dx.doi.org/10.1590/1413-81232018234.09962016> doi: 10.1590/141381232018234.09962016

ACUNA, K.; CRUZ, T. Avaliação do estado nutricional de adultos e idosos e situação nutricional da população brasileira. Arquivos Brasileiros de Endocrinologia e $\begin{array}{llll}\text { Metabologia, } \quad \text { v.48, } & \text { n.3, } 2004 . \quad \text { Disponivel }\end{array}$ em:<https://doi.org/10.1590/S0004-27302004000300004> doi: 10.1590/S000427302004000300004.

ARRUDA, N. B. M.,SILVA, L.M.L.; ARAUJO, NA.C.R.; SILVA, A.E.A.O.; ANGELIM, M.K.S.C. et al. Associação entre o tempo de internação e indicadores de massa muscular em idosos hospitalizados. Brazilian Journal of Develop, v. 6, n. 4, p. 21832-21847, 2020.

AZEVEDO, L. C. FENILLI, M.; NEVES, L.; FARIAS, M.B.; BREITKOPF, T.S. et al. Principais fatores da mini-avaliação nutricional associada a alterações nutricionais de idosos hospitalizados. Arquivos Catarinenses de Medicina, v. 36, n. 3, p. 7-14, 2007.

BARBOSA, A. A. DE O.; VICENTINI, A. P.; LANGA, F. R. Comparação dos critérios da nrs-2002 com o risco nutricional em pacientes hospitalizados. Ciência e saúde coletiva, v.24, n.9, 2019. Disponível em: <https://doi.org/10.1590/141381232018249.25042017> doi: 10.1590/1413-81232018249.25042017

BHIROMMUANG N, KOMINDR S, JAYANAMA K. Impact of nutritional status on length of stay and hospital costs among patients admitted to a tertiary care hospital in Thailand. Asia Pacific Journal of Clinical Nutrition, v.28, n. 2, p.252-259, 2019. Disponível em: <http://dx.doi.org/10.6133/apjcn.201906_28(2).0007> doi:10.6133/apjcn.201906_28(2).0007

CAMARGOS, M.C.S.; GONZAGA, M. R.; COSTA, J. V.; BOMFIM, W.C.; Estimativas de expectativa de vida livre de incapacidade funcional para Brasil e Grandes Regiões, 1998 e 2013. Ciência \& Saúde Coletiva, v. 24, n.3, p. 737-747, 2019. Disponível em: <https://doi.org/10.1590/1413-81232018243.07612017> doi: 10.1590/1413-81232018243.07612017

CENDRON, G da SILVA, L. J.; CLOSS, V. E. SCHWANKE, C. H. A.; EL KIK, R. Estado Nutricional e Capacidade Funcional em idosos hospitalizados. Revista da Graduação, v. 9, n. 1, 2016. 
CHAN, S. P.; IP, K. Y.; IRWIN, M. G. Peri-operative optimisation of elderly and frail patients: a narrative review. Anaesthesia ,v. 74, n. 1, p. 80-89, 2019. Disponível em: <https://doi.org/10.1111/anae.14512> doi: doi:10.1111/anae.14512

CHUMLEA, W.M.C.; GUO, S.S.; ROCHE, A.F.; et al. Prediction of body weight for the nonambulatory elderly from anthropometry. Journal of the American Dietetic Association, v.88, n.5, p. 564-8, 1988.

CRUZ, A. P. M.; PINTO, L. R. C.; LAGE, Y. G.; NASRALA, M.L.S.; NASRALA NETO, E.; Alterações da capacidade funcional de idosos durante a internação hospitalar. COORTE - Revista Científica do Hospital Santa Rosa. Cuiabá-MT, v.3, p. 22-29, 2011.

DIETRICH, C.; CARDOSO, J. R.; VARGAS, F.; SANCHEZ, E.C.; DUTRA, F.H.; MOREIRA, C. et al. Capacidade funcional em idosos e idosos mais velhos após alta da unidade de terapia intensiva. Coorte prospectiva. Revista Brasileira de Terapia Intensiva, v. 29, n. 3, p.293-302, 2017. Disponível em: https://doi.org/10.5935/0103507X.20170055> doi: 10.1590/S0102-311X2012001100008

FREITAS, F. A. S.; SANTOS, E. S. DE S.: PEREIRA, L. S. M.; LUSTOSA, L.P. ; Vulnerabilidade física de idosos na alta hospitalar. Fisioterapia e Pesquisa, v.24, n. 3. p. 253-258, 2017. Disponível em: <http://dx.doi.org/10.1590/18092950/16205224032017> doi: 10.1590/1809-2950/16205224032017

FRISANCHO, A. R. Anthropometric Standards for the Assessment of Growth and Nutritional Status. Ann Arbor, Michigan: University of Michigan Press, 1990.

GURNEY, J.M., JELLIFFE, D.B. Arm anthropometry in nutritional assessment: nomogram for rapid calculation of muscle circumference and crosssectional muscle and fat areas. The American Journal of Clinical Nutrition, v. 26, p. 912-915, 1973.

HEYMSFIELD, S.B.; MCMANUS, C.; SMITH, J.; STEVENS, V.; NIXON, D.W.; Anthropometric measurement of muscle mass: revised equations for calculating bone-free arm muscle area. The American Journal of Clinical Nutrition, v. 36, n.4, p.680-690, 1982. Disponivel em: <https://doi.org/10.1093/ajcn/36.4.680> doi:10.1093/ajcn/36.4.680>

KATSAS, K.; MAMALAKI, E.; KONTOGIANNI, M.D.; ANASTASIOU, C.A.; Malnutrition in older adults: correlations with social, diet-related and neuropsychological. Nutrition, 110640, 2019. Disponível em:< https://doi.org/10.1016/j.nut.2019.110640> doi: 10.1016 / j.nut.2019.110640

KIM, T. N., CHOI, K. M. The implications of sarcopenia and sarcopenic obesity on cardiometabolic disease. J Cell Biochem, v. 116, n. 7, p. 1171-8, 2015. Disponível em: <https://doi.org/10.1002/jcb.25077> doi:10.1002/jcb.25077

LEITE, H. P.; CARVALHO, W. B.; SANTANA E MENESES, J. F. Atuação da equipe multidisciplinar na terapia nutricional de pacientes sob cuidados intensivos. Revista de Nutrição, v.18, n.6, 2005. Disponível em: <https://doi.org/10.1590/S141552732005000600008> doi:10.1590/S1415-52732005000600008 
LOURO, C. A. S. Avaliação do estado nutricional do idoso hospitalizado. 83 f. Trabalho de Investigação - Faculdade Ciências de Nutrição e Alimentação, Universidade do Porto, 2004.

MIRANDA, G. M.; MENDES, A. DA C. G.; DA SILVA, A. L. A. O envelhecimento populacional brasileiro: desafios e consequências sociais atuais e futuras. Revista brasileira de geriatria e gerontologia, v.19, n.3, 2016. Disponivel em: <http://dx.doi.org/10.1590/1809-98232016019.150140>doi: 10.1590/180998232016019.150140

MISHAMANDANI, Z. J.; NOROUZY, A.; HASHEMIAN, S.M.; KHOUNDABI, H.; REZAEISADRABADI, $M$. et al. Nutritional status of patients hospitalized in the intensive care unit: A comprehensive report from Iranian hospitals, 2018. Journal of Critical Care, v.54, p. 151-158, 2019. Disponível em: <http://dx.doi.org/10.1016 / j.jcrc.2019.08.001 > doi: 10.1016 / j.jcrc.2019.08.001

NAJAS, M.; YAMATTO, T.H. Avaliação do estado nutricional de idosos. Nestlé Nutricion. Disponível: $<$ http://www.nestlenutricaodomiciliar.com.br/Files/documentos/AVALIACAO\%20ESR\%20NUT>. Acesso em: 10 abr. 2018.

OLIVEIRA, F. M. R. L. DE; COSTA, K. N. DE F. M.; PONTES, M. DE L. DE F.; BATISTA, P.S.S.; BARBOSA, K.T.F., et al. Fatores de risco associados à hospitalização em idosos atendidos na atenção primária de saúde. Revista de enfermagem UERJ, v. 26, p. 15488, 2018. Disponível em: <http://dx.doi.org/10.12957/reuerj.2018.15488> doi:10.12957/reuerj.2018.15488

OLIVEIRA, K.D. L.; HAACK, A.; FORTES, R. C. Estado nutricional de idosos e prevalência de lesão por pressão na assistência domiciliar. Revista Enfermagem Atual, Edição Especial, 2017. Disponível em:<https://doi.org/10.31011/reaid-2017v.2017-n.0-art.551> doi: https://doi.org/10.31011/reaid-2017-v.2017-n.0-art.551

Organización Panamericana de la Salud. División de Promoción y Protección de la Salud (HPP). Encuesta Multicentrica salud beinestar y envejecimiento (SABE) em América Latina el Caribe: Informe Preliminar. XXXVI Reunión del Comité asesor de investigaciones em Salud; 9-11 jun 2001; Kingston, Jamaica: OPAS, 2002 [acesso em 14 abril 2020]. Disponível em: www.opas.org/program/sabe.htm.

PANISSA, C. O.; VASSIMON, H. S. Risco de desnutrição de idosos hospitalizados: avaliando ingestão alimentar e antropometria. DEMETRA: Alimentação, Nutrição \& Saúde, v. $7, \quad$ n. 1, p. 13-22, 2012. Disponível em: <http://dx.doi.org/10.12957/demetra.2012.3275> doi:10.12957/demetra.2012.3275

RIBEIRO, I. A.; LIMA, L.R.; VOLPE, C.R.G.; FUNGHETTO, S.S.; REHEM, T.C.M.S.B et al. Síndrome do idoso frágil em idosos com doenças crônicas na Atenção Primária. Revista da Escola de Enfermagem da USP, v. 53, e03449, 2019.

SAMPAIO, L.S.; CARNEIRO, J.A.O.; COQUEIRO, R.S.; FERNANDES, M.H. Indicadores antropométricos como preditores na determinação da fragilidade em idosos. Ciência \&Saúde Coletiva, v. 22, n.12, p.4115-4123, 2017. 
SHUHADA, N. A.; AZIZ, A.; MOHD, N. I.; ISMAIL, N.H.; Assessing the nutritional status of hospitalized elderly. Clinical Interventions in Aging, v. 12, p. 1615-1625, 2017. Disponível em:<https://doi.org/10.2147/CIA.S140859> doi: 10.2147/CIA.S140859

SILVA, V. C.; SANTO, F. H. do E.; PEREIRA, E. R.; SILVA, J.; SILVA, J.S.; SANTOS, L.; Acompanhamento da capacidade funcional de idosos hospitalizados. Caderno de Saúde Pública, v.34, n.11, p.00173317, 2018. Disponivel em: <http://dx.doi.org/10.23925/2176-901X.2019v22i4p245-263 > doi: 10.23925/2176901X.2019v22i4p245-263

SILVA, G. A.; SILVA, G. D. da.; CABRAL, A. M. B. de O.; SANTIAGO, E.R.C.; ARAUJO, E.R.S. et al.; Estado nutricional e capacidade funcional de idosos oncológicos hospitalizados. Nutrición clínica y dietética hospitalaria, v. 39, n.2, p. 54-62, 2019. Disponível em: <https://dx.doi.org/10.12873/392gabriela> doi: 10.12873/392gabriela

SOUSA, J. C. S.; DE OLIVEIRA, C. M.; ARAÚJO, A. S. M.; SANTO, R.D.; Avaliação do estado nutricional de pacientes hospitalizados: uso de métodos convencionais e não convencionais. Demetra, v. 13, n.1, p. 195-208, 2018. Disponível em: <https://doi.org/ 10.12957/demetra.2018.29094> doi: 10.12957/demetra.2018.29094

STATSOFT, Inc. (2011). STATISTICA (data analysis software system), version 10.

TAVARES, E.L.; dos SANTOS, D. M; FERREIRA, A. A.; MENEZES, M.F.G.; Avaliação nutricional de idosos: desafios da atualidade. Revista Brasileira de Geriatria e Gerontologia, v.18, n.3, 2015. Disponível em: <http://dx.doi.org/10.1590/1809-9823.2015.14249> doi:10.1590/18099823.2015.14249

TEIXEIRA, V. P.; DE MIRANDA, R. C.; BAPTISTA, D. R. Desnutrição na admissão, permanência hospitalar e mortalidade de pacientes internados em um hospital terciário. Demetra, v. 11, n. 1, p. 239-251, 2016. Disponível em: <https://doi.org/10.12957/demetra.2016.18457> doi: 10.12957/demetra.2016.18457

The Hartford Institute for Geriatric Nursing. Katz Index of Independence in Activities of Daily Living (ADL) [1998]. New York; 2005. [acessado em 14 de abril de 2020]. Available from: http://www.hartfordign.org/

WOODROW, G. Body composition analysis techniques in the aged adult: indications and limitations. Curr Opin Clin Nutr Metab Care, v. 12, n. 1, p. 8-14, 2009.

ZANCHIM, M. C.; LIBERALI, R.; COUTINHO, V. Estado nutricional de idosos hospitalizados em um hospital geral de alta complexidade do Estado do Rio Grande do Sul. Revista brasileira de nutrição clínica, v. 28, n.4, p. 292-9, 2013. 